open ${ }^{\text {DE gruter }}$ ACta Univ. SAPientiae, Informatica 8, 1 (2016) 96-107

DOI: 10.1515/ausi-2016-0006

\title{
On the nullity of a family of tripartite graphs
}

\author{
Rashid FAROOQ \\ School of Natural Sciences, \\ National University of Sciences and \\ Technology, Islamabad, Pakistan \\ email: farook.ra@gmail.com

\section{Qudsia NAUREEN} \\ School of Natural Sciences, \\ National University of Sciences and \\ Technology, Islamabad, Pakistan \\ email: naureenqudsia@gmail.com
}

\author{
Mehar Ali MALIK \\ School of Natural Sciences, \\ National University of Sciences and \\ Technology, Islamabad, Pakistan \\ email: alies. camp@gmail.com \\ Shariefuddin PIRZADA \\ University of Kashmir, Srinagar, India \\ email: \\ pirzadasd@kashmiruniversity.ac.in
}

\begin{abstract}
The eigenvalues of the adjacency matrix of a graph form the spectrum of the graph. The multiplicity of the eigenvalue zero in the spectrum of a graph is called nullity of the graph. Fan and Qian (2009) obtained the nullity set of $n$-vertex bipartite graphs and characterized the bipartite graphs with nullity $n-4$ and the regular $n$-vertex bipartite graphs with nullity $n-6$. In this paper, we study similar problem for a class of tripartite graphs. As observed the nullity problem in tripartite graphs does not follow as an extension to that of the nullity of bipartite graphs, this makes the study of nullity in tripartite graphs interesting. In this direction, we obtain the nullity set of a class of $n$-vertex tripartite graphs and characterize these tripartite graphs with nullity $n-4$. We also characterize some tripartite graphs with nullity $n-6$ in this class.
\end{abstract}

Computing Classification System 1998: G.2.2

Mathematics Subject Classification 2010: 05C50

Key words and phrases: nullity, tripartite graph, expanded path 


\section{Introduction}

Let $G$ be a simple graph with vertex set $V(G)=\left\{v_{1}, \ldots, v_{n}\right\}$ and edge set $\mathrm{E}(\mathrm{G})$. An edge joining a vertex $v_{i}$ to a vertex $v_{j}$ is denoted by $v_{i} v_{j}\left(\right.$ or $\left.v_{j} v_{i}\right)$. The adjacency matrix of $\mathrm{G}$ is $\mathrm{A}(\mathrm{G})=\left[\mathrm{a}_{\mathrm{ij}}\right]_{\mathfrak{n} \times \mathfrak{n}}$, where

$$
a_{i j}=\left\{\begin{array}{ll}
1 & \text { if } v_{i} v_{j} \in E(G) \\
0 & \text { otherwise }
\end{array} \quad\left(\forall v_{i}, v_{j} \in V(G)\right)\right.
$$

The eigenvalues of the graph $G$ are the eigenvalues of $A(G)$ and the spectrum of $\mathrm{G}$ is the multiset of eigenvalues of $\mathrm{G}$. The nullity of graph $\mathrm{G}$, denoted by $\eta(G)$, is the multiplicity of the eigenvalue zero in the spectrum of $G$. The graph $G$ is singular if $\eta(G)>0$ and is non-singular if $\eta(G)=0$. For graph theoretical terminology, we refer [10].

Collatz and Sinogowitz [2] posed the problem of characterizing singular graphs and since then, the theory of nullity of graphs has stimulated much research because of its importance in mathematics and chemistry. In literature, we find characterization of trees, unicyclic graphs, bicyclic graphs and bipartite graphs with their nullity.

Fiorini et al. [5] determined the greatest nullity among n-vertex trees in which no vertex has degree greater than a fixed positive integer D and gave a method of constructing the respective trees. Li and Chang [7] showed that there are some other trees with maximum nullity which can not be constructed by the method devised by Fiorini et al. [5] and modified the method of constructing the respective trees. Tan and Liu [11] found the nullity set of $n$-vertex unicyclic graphs with $n \geq 5$. They also characterized the unicyclic graphs with maximal nullity. The unicyclic graphs with minimum nullity are characterized by $\mathrm{Li}$ and Chang [8]. Li et al. [9] found the nullity set of $n$-vertex bicyclic graphs and gave a characterization of the bicyclic graphs with maximum nullity. Hu et al. [6] also found the nullity set of $n$-vertex bicyclic graphs, $n \geq 6$. Furthermore, the authors also characterized bicyclic graphs with extremal nullity. In Fan et al. [3], the authors found the nullity of unicyclic signed graphs and characterized n-vertex unicyclic signed graphs. Fan and Qian [4] introduced nullity of $n$-vertex bipartite graphs and presented characterization of bipartite graphs with nullity.

We find that the nullity of tripartite graphs does not follow as the extension of that of bipartite graphs. Thus it became interesting to characterize family of tripartite graphs with nullity. In this paper, we consider a class of n-vertex tripartite graphs, $n \geq 3$ and give nullity set of these tripartite graphs and characterize them with nullity $n-4, n \geq 4$. Furthermore, we discuss some tripartite graphs with nullity $n-6, n \geq 6$. 


\section{Preliminaries}

In this section, we give some definitions, terminologies and known results which will be used later. Let $G$ be an n-vertex graph with vertex set $V(G)$ and edge set $E(G)$. For $S \subseteq V(G)$, the neighbor set of $S$ in $G$, denoted as $N(S)$, is a set containing those vertices of $G$ that are adjacent to some vertex in $S$. If $S=\{v\}$, we write $N(v)$ for $N(\{v\})$. If $S$ is nonempty, we denote by $G[S]$ the induced subgraph of $\mathrm{G}$. The $\operatorname{rank}$ of the graph $\mathrm{G}$, denoted by $\operatorname{rank}(\mathrm{G})$, is the $\operatorname{rank}$ of its adjacency matrix $A(G)$, that is, $\operatorname{rank}(G)=\operatorname{rank}(A(G))$. It is known that $\eta(G)=n-\operatorname{rank}(G)$. The graph $G$ is said to be an expanded graph if its vertex set $V(G)$ can be partitioned into $V_{1}, V_{2} \ldots, V_{k}, k \geq 2$, such that $G\left[V_{i}\right]$ is an empty graph, for $1 \leq i \leq k$. If $G\left[V_{i} \cup V_{j}\right]$ is a nonempty graph, it is a complete bipartite graph for $1 \leq i, j \leq k, i \neq j$. If $G$ is an expanded graph on $V_{1}, V_{2}, \ldots, V_{k}$, each $V_{i}$, for $1 \leq i \leq k$, is called an expanded vertex of order $\left|V_{i}\right|$. We observe that each simple graph can be viewed as an expanded graph.

The $n$-vertex graph $\mathrm{G}$ is said to be an expanded path of length $k$ if its vertex set $\mathrm{V}(\mathrm{G})$ can be partitioned into $\mathrm{V}_{1}, \ldots, \mathrm{V}_{\mathrm{k}}, \mathrm{k} \geq 2$, such that

(i) $G\left[V_{i}\right]$ is an empty graph for $1 \leq i \leq k$,

(ii) $G\left[V_{i} \cup V_{i+1}\right]$ is a complete bipartite graph for $1 \leq i \leq k-1$,

(iii) $G\left[V_{i} \cup V_{j}\right]$ is an empty graph for $1 \leq i, j \leq k$ with $j \neq i+1$.

We use the notation $\mathbb{P}_{k}\left(V_{1}, \ldots, V_{k}\right)$ to denote an expanded path on $V_{1}, \ldots, V_{k}$ of length $k$. Similarly, an expanded cycle of length $k$, denoted by $\mathbb{C}_{k}\left(V_{1}, \ldots, V_{k}\right)$, is obtained from the expanded path $\mathbb{P}_{k}\left(V_{1}, \ldots, V_{k}\right)$ by adding edges between each vertex of $V_{1}$ and each of $V_{k}$. When there is no ambiguity, we simply write $\mathbb{P}_{k}$ and $\mathbb{C}_{k}$ respectively to represent an expanded path and an expanded cycle of length $\mathrm{k}$. An expanded decomposition of the graph $\mathrm{G}$ is a list of expanded subgraphs such that each edge of $G$ appears in exactly one expanded subgraph in the list.

The graph $G$ is tripartite if its vertex set can be partitioned into three subsets $X, Y$ and $Z$ such that $G[X], G[Y]$ and $G[Z]$ are empty graphs; such a partition $(X, Y, Z)$ is called a tripartition. For any $S \subseteq V(G)$, we denote by $N_{X}(S)$ the neighbors of $S$ in $X$. Analogously, we can define $N_{Y}(S)$ and $N_{Z}(S)$. We consider a special class of tripartite graphs defined as follows. Let $\mathcal{T}_{n}$ be the family of those $n$-vertex tripartite graphs $G, n \geq 5$, whose tripartition $(X, Y, Z)$ satisfies the following:

$$
\begin{aligned}
& \mathrm{N}_{X}\left(\mathrm{Y}^{\prime}\right) \neq \mathrm{X} \text { and } \mathrm{N}_{\mathrm{Z}}\left(\mathrm{Y}^{\prime}\right) \neq \mathrm{Z} \forall \mathrm{Y}^{\prime} \subseteq \mathrm{Y} \\
& \mathrm{G}[\mathrm{X} \cup \mathrm{Z}] \text { is complete bipartite. }
\end{aligned}
$$


Lemma 1 (Fan [4]) An expanded path of length $\mathrm{k} \geq 2$ and order $\mathrm{n}$ has nullity $\mathrm{n}-\mathrm{k}$, if $\mathrm{k}$ is even and $\mathrm{n}-\mathrm{k}+1$, if $\mathrm{k}$ is odd.

Let $K_{n_{1}, n_{2}}$ denote the complete bipartite graph, where $n_{1}$ and $n_{2}$ are the sizes of its partite sets. Also, $K_{1}$ denotes an isolated vertex and $r K_{1}$ denotes $r$ copies of $K_{1}$. Then the following result characterizes the graphs with nullity $n-2$.

Lemma 2 (Cheng [1]) Let $\mathrm{G}$ be an $\mathrm{n}$-vertex simple graph, $\mathrm{n} \geq 2$. Then $\eta(G)=n-2$ if and only if $\mathrm{G} \cong \mathrm{K}_{n_{1}, n_{2}} \cup \mathrm{n}_{3} \mathrm{~K}_{1}$, where $\mathrm{n}=\mathrm{n}_{1}+\mathrm{n}_{2}+\mathrm{n}_{3}$, $\mathrm{n}_{1}, \mathrm{n}_{2}>0$ and $\mathrm{n}_{3} \geq 0$.

\section{Main results}

Let $\mathrm{G} \in \mathcal{T}_{\mathrm{n}}$ with tripartition $(\mathrm{X}, \mathrm{Y}, \mathrm{Z})$. The adjacency matrix $A(\mathrm{G})$ of $\mathrm{G}$ is defined by

$$
\left.A(G)=\underset{Z}{X} \begin{array}{ccc}
X & Z & Y \\
\hline & J & C_{1} \\
J^{t} & 0 & C_{2} \\
C_{1}^{t} & C_{2}^{t} & 0
\end{array}\right],
$$

where $\mathrm{J}$ and $\mathbf{0}$ respectively denote the matrices with all entries 1 and 0 . Let $\mathrm{C}$ and $\mathrm{B}$ denote the matrices defined as follows.

$$
\mathrm{C}=\left[\begin{array}{l}
\mathrm{C}_{1} \\
\mathrm{C}_{2}
\end{array}\right] \quad \text { and } \quad \mathrm{B}=\left[\begin{array}{cc}
\mathbf{0} & \mathrm{J} \\
\mathrm{J}^{\mathrm{t}} & \mathbf{0}
\end{array}\right]
$$

The matrix $A(G)$ can be viewed as

$$
A(G)=\left[\begin{array}{cc}
B & C \\
C^{t} & 0
\end{array}\right]
$$

Let

$$
\mathrm{U}=\left[\begin{array}{ll}
\mathrm{B} & \mathrm{C}
\end{array}\right], \quad \mathrm{L}=\left[\begin{array}{ll}
\mathrm{C}^{\mathrm{t}} & 0
\end{array}\right] .
$$

Then $A(G)$ can be written as $A(G)=\left[\begin{array}{l}U \\ L\end{array}\right]$.

For each $v \in X \cup Z$, we denote by $U_{v}$ the row of $A(G)$ corresponding to the vertex $v$. Similarly, for each $v \in Y$, the row of $A(G)$ corresponding to the 
vertex $v$ is denoted by $L_{v}$. Let $S \subseteq X \cup Z$. Then from the matrix $A(G)$, we see that

$$
\sum_{v \in S} k_{v} u_{v}=\left[\begin{array}{lll}
b_{1} & b_{2} & c
\end{array}\right]
$$

where $b_{1}, b_{2}$ are constant row matrices respectively of dimension $1 \times|X|$ and $1 \times|Z|, c$ is row vector of dimension $1 \times|Y|$, and $k_{v}$ 's are real constants. Similarly, for any $Y^{\prime} \subseteq Y$, we can write

$$
\sum_{v \in Y^{\prime}} k_{v}^{\prime} L_{v}=\left[\begin{array}{lll}
c_{1} & c_{2} & 0
\end{array}\right]
$$

where $c_{1}, c_{2}$ and $\mathbf{0}$ are row vectors respectively of dimension $1 \times|X|, 1 \times|Z|$ and $1 \times|\mathrm{Y}|$, and $k_{v}^{\prime}$ 's are real constants.

The following result gives information about the rank of a tripartite graph in $\mathcal{T}_{\mathrm{n}}$.

Lemma 3 Let $\mathrm{G} \in \mathcal{T}_{\mathrm{n}}$ with tripartition $(\mathrm{X}, \mathrm{Y}, \mathrm{Z})$ and adjacency matrix defined by (3). Then

$$
\operatorname{rank}(\mathrm{G})=\operatorname{rank}(\mathrm{U})+\operatorname{rank}(\mathrm{L}),
$$

where $\mathrm{U}$ and $\mathrm{L}$ are defined by (4).

Proof. Let $S$ and $Y^{\prime}$ be arbitrary subsets, respectively of $X \cup Z$ and $Y$. To prove (7), it is enough to show that $\sum_{v \in S} k_{v} \mathrm{U}_{v} \neq \sum_{v \in Y^{\prime}} k_{v}^{\prime} \mathrm{L}_{v}$ whenever $\sum_{v \in S} k_{v} \mathrm{U}_{v} \neq$ 0 and $\sum_{v \in Y^{\prime}} k_{v}^{\prime} \mathrm{L}_{v} \neq 0$, and $k_{v}$ 's and $k_{v}^{\prime}$ 's are real constants.

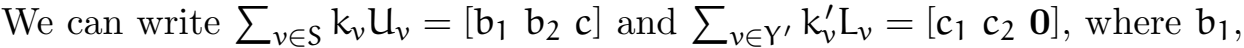
$\mathrm{b}_{2}, \mathrm{c}, \mathrm{c}_{1}, \mathrm{c}_{2}$ and $\mathbf{0}$ are defined in (5) and (6). By condition (1), there exists a vertex in $X$ which is not adjacent to any vertex in $Y$. Similarly, there exists a vertex in $Z$ which is not adjacent to any vertex in $Y$. Thus there are at least two zero columns in $C^{t}$ corresponding to a vertex in $X$ and to a vertex in $Z$. That is, there are zero entries in vectors $c_{1}$ and $c_{2}$. Now, if $\sum_{v \in S} k_{v} U_{v}=\sum_{v \in Y^{\prime}} k_{v}^{\prime} L_{v}$ then $\left[\begin{array}{lll}b_{1} & b_{2} & c\end{array}\right]=\left[\begin{array}{lll}c_{1} & c_{2} & 0\end{array}\right]$. As $b_{1}$ and $b_{2}$ are constant vectors, the vectors $b_{1}$, $b_{2}, c, c_{1}, c_{2}$ are all zero vectors. This completes the proof.

Corollary 4 Let $\mathrm{G} \in \mathcal{T}_{\mathrm{n}}$ with tripartition $(\mathrm{X}, \mathrm{Y}, \mathrm{Z})$ and the adjacency matrix $\mathrm{A}(\mathrm{G})$ defined by $(3)$. Then $\operatorname{rank}(\mathrm{G})=2(1+\operatorname{rank}(\mathrm{C}))$.

Proof. By the construction of the matrix $A(G)$ and by the arguments used in Lemma 3, we see that $\operatorname{rank}(U)=\operatorname{rank}(B)+\operatorname{rank}(C)$. Since $\operatorname{rank}(B)=2$ and $\operatorname{rank}(\mathrm{L})=\operatorname{rank}(\mathrm{C})=\operatorname{rank}\left(\mathrm{C}^{\mathrm{t}}\right)$, we get from $(7)$ that $\operatorname{rank}(\mathrm{G})=2(1+$ $\operatorname{rank}(\mathrm{C}))$. 
Let $\mathbb{C}_{k}(\bar{e})$ denote an expanded cycle of length $k$ with an expanded chord $\bar{e}$ joining two non-adjacent expanded vertices of the cycle $\mathbb{C}_{k}$. We have the following observation.

Lemma 5 If $\mathrm{G}=\mathbb{C}_{5}(\overline{\mathrm{e}}) \cup \mathrm{kK}_{1}$ is a graph of order $\mathrm{n}$ shown in Figure $1, \mathrm{k} \geq 0$, then $\mathrm{G} \in \mathcal{T}_{\mathrm{n}}$ and $\eta(\mathrm{G})=\mathrm{n}-4$.

Proof. Let $X=X_{1} \cup X^{\prime}, Z=Z_{1} \cup Z^{\prime}$ and $Y=Y_{1} \cup Y^{\prime}$, where $Y^{\prime}$ is possibly empty. Then we see that the graph $G$ is a tripartite graph with tripartition $(X, Y, Z)$. Moreover, $G$ satisfies (1) because $N_{X^{\prime}}(Y)=\emptyset$ and $N_{Z^{\prime}}(Y)=\emptyset$. Also, $\mathrm{G}[\mathrm{X} \cup \mathrm{Z}]=\mathbb{P}(\mathrm{X}, \mathrm{Z})$, that is, $\mathrm{G}$ satisfies (2). Thus $\mathrm{G} \in \mathcal{T}_{\mathrm{n}}$. Let $A(\mathrm{G})$ be the adjacency matrix of $G$ defined by (3). By the construction of $G$, we see that all rows of $\mathrm{C}^{\mathrm{t}}$ are identical and therefore $\operatorname{rank}(\mathrm{C})=1$. By Corollary 4 , we conclude that $\eta(G)=n-4$.

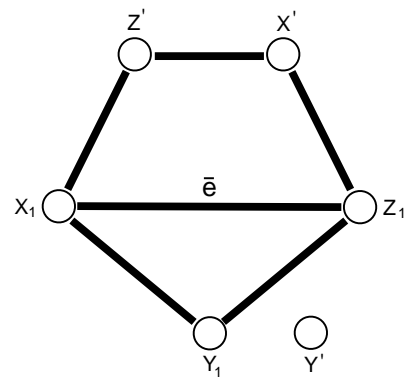

Figure 1: An expanded graph $\mathbb{C}_{5}(\bar{e}) \cup \mathrm{kK}_{1}$

The next result gives the nullity set of the graphs in $\mathcal{T}_{n}$.

Theorem 6 Let $\mathrm{m}_{1}, \mathrm{~m}_{2}$ and $\mathrm{m}_{3}$ be positive integers such that $\mathrm{n}=\mathrm{m}_{1}+\mathrm{m}_{2}+$ $\mathrm{m}_{3}$. Then for each integer $\mathrm{k} \in\left\{0,1, \ldots, \min \left\{\mathrm{m}_{1}+\mathrm{m}_{3}-2, \mathrm{~m}_{2}\right\}\right\}$, there is a graph $\mathrm{G} \in \mathcal{T}_{\mathrm{n}}$ with tripartition $(\mathrm{X}, \mathrm{Y}, \mathrm{Z})$ such that $|\mathrm{X}|=\mathrm{m}_{1},|\mathrm{Y}|=\mathrm{m}_{2},|\mathrm{Z}|=\mathrm{m}_{3}$ and $\eta(G)=n-2(k+1)$. Conversely, if $G \in \mathcal{T}_{\mathfrak{n}}$ with tripartition $(X, Y, Z)$ then $\eta(G)=n-2(1+k)$, where $k \in\{0,1, \ldots, \min \{|X|+|Z|-2,|Y|\}\}$.

Proof. First, we prove that for each $k \in\left\{0,1, \ldots, \min \left\{m_{1}+m_{3}-2, m_{2}\right\}\right\}$, there is a graph $G \in \mathcal{T}_{\mathfrak{n}}$ with tripartition $(X, Y, Z)$ such that $|X|=m_{1},|Y|=m_{2},|Z|=$ $m_{3}$ and $\eta(G)=n-2(k+1)$. We take three non-empty sets $X=\left\{x_{1}, x_{2}, \ldots, x_{m_{1}}\right\}$, $Y=\left\{y_{1}, y_{2}, \ldots, y_{m_{2}}\right\}$ and $Z=\left\{z_{1}, z_{2}, \ldots, z_{m_{3}}\right\}$. If $k=0$, we construct a graph $\mathrm{G}=\mathrm{P}(\mathrm{X}, \mathrm{Z}) \cup \mathrm{m}_{2} \mathrm{~K}_{1}$. Clearly, $\mathrm{G} \in \mathcal{T}_{\mathrm{n}}$. By using Lemma $2, \eta(\mathrm{G})=\mathrm{n}-2$. If $k>0$, we consider following two cases. 
Case 1. When $k \leq m_{1}-1$. Since $k \leq m_{2}$, we construct a tripartite graph $G$ with tripartition $(X, Y, Z)$ that satisfies the following.

(i) $\mathrm{G}[\mathrm{X} \cup \mathrm{Z}]=\mathbb{P}(\mathrm{X}, \mathrm{Z})$,

(ii) $\left|N_{X}\left(y_{i}\right)\right|=1$ and $N_{z}\left(y_{i}\right)=\emptyset$ for $1 \leq i \leq k$,

(iii) $N_{X}\left(y_{i}\right) \cap N_{X}\left(y_{j}\right)=\emptyset$ for $i \neq j$ and $1 \leq i, j \leq k$,

(iv) $d\left(y_{i}\right)=0$ for $k+1 \leq i \leq m_{2}$.

Then $\mathrm{G} \in \mathcal{T}_{\mathrm{n}}$. Moreover, the adjacency matrix of $\mathrm{G}$ is given by (3), where

$$
\mathrm{C}=\left[\begin{array}{l:l}
\mathrm{I}_{\mathrm{k} \times \mathrm{k}} & \mathbf{0}_{\mathrm{k} \times\left(\mathrm{m}_{2}-\mathrm{k}\right)} \\
\hdashline \mathbf{0}_{\left(\mathrm{m}_{1}+\mathrm{m}_{3}-\mathrm{k}\right) \times \mathrm{k}} & \mathbf{0}_{\left(\mathrm{m}_{1}+\mathrm{m}_{3}-\mathrm{k}\right) \times\left(\mathrm{m}_{2}-\mathrm{k}\right)}^{-}
\end{array}\right] .
$$

Then $\operatorname{rank}(C)=k$. By Corollary 4 , we get $\eta(G)=n-2(1+k)$.

Case 2. When $k>m_{1}-1$. Since $k \leq m_{2}$ and $k-\left(m_{1}-1\right) \leq m_{3}-1$, we construct a tripartite graph $G$ with tripartition $(X, Y, Z)$ that satisfies the following.

(i) $\mathrm{G}[\mathrm{X} \cup \mathrm{Z}]=\mathbb{P}(\mathrm{X}, \mathrm{Z})$,

(ii) $\left|\mathrm{N}_{X}\left(\mathrm{y}_{\mathrm{i}}\right)\right|=1$ for $1 \leq \mathrm{i} \leq \mathrm{m}_{1}-1$,

(iii) $\left|N_{z}\left(y_{i}\right)\right|=1$ for $m_{1} \leq i \leq k$,

(iv) $N_{X}\left(y_{i}\right) \cap N_{X}\left(y_{j}\right)=\emptyset$ for $i \neq j$ and $1 \leq i, j \leq m_{1}-1$,

(v) $N_{Z}\left(y_{i}\right) \cap N_{Z}\left(y_{j}\right)=\emptyset$ for $i \neq j$ and $m_{1} \leq i, j \leq k$,

(vi) $\mathrm{d}\left(\mathrm{y}_{\mathrm{i}}\right)=0$ for $\mathrm{k}+1 \leq \mathrm{i} \leq \mathrm{m}_{2}$.

Then $\mathrm{G} \in \mathcal{T}_{\mathrm{n}}$. Moreover, the adjacency matrix of $\mathrm{G}$ is given by (3), where

$$
\mathrm{C}=
$$

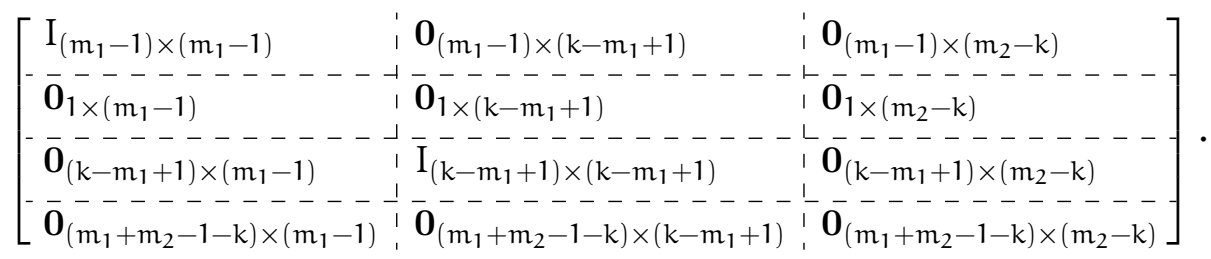

Then $\operatorname{rank}(C)=k$. Corollary 4 gives $\eta(G)=n-2(1+k)$. 
Conversely, we show that if $G \in \mathcal{T}_{\mathfrak{n}}$ with tripartition $(X, Y, Z)$, then $\eta(G)=$ $n-2(1+k)$ where $k \in\{0,1, \ldots, \min \{|X|+|Z|-2,|Y|\}\}$. By Corollary $4, \operatorname{rank}(G)=$ $2(1+\operatorname{rank}(C))$. By $(1)$, there are at least two zero rows in $C$. This implies that $\operatorname{rank}(\mathrm{C}) \leq \min \{|X|+|\mathrm{Z}|-2,|\mathrm{Y}|\}$. The result is true by setting $\operatorname{rank}(\mathrm{C})=\mathrm{k}$.

From Corollary 4, for each graph $\mathrm{G} \in \mathcal{T}_{\mathrm{n}}$ with $\mathrm{A}(\mathrm{G})$ defined by (3), we can write

$$
\eta(G)=n-2(1+\operatorname{rank}(C)) .
$$

The next result is a direct consequence of Lemma 2.

Theorem 7 For a graph $\mathrm{G} \in \mathcal{T}_{\mathrm{n}}$ with tripartition $(\mathrm{X}, \mathrm{Y}, \mathrm{Z}), \mathrm{\eta}(\mathrm{G})=\mathrm{n}-2$ if and only if $\mathrm{G}=\mathbb{P}(\mathrm{X}, \mathrm{Z}) \cup|\mathrm{Y}| \mathrm{K}_{1}$.

Proof. Let $G \in \mathcal{T}_{n}$ with tripartition $(X, Y, Z)$ and $\eta(G)=n-2$. Then from equation (8), we have $\operatorname{rank}(C)=0$. That is, $d(y)=0$ for all $y \in Y$. Thus $\mathrm{G}=\mathbb{P}(\mathrm{X}, \mathrm{Z}) \cup|\mathrm{Y}| \mathrm{K}_{1}$. Conversely, suppose that $\mathrm{G}=\mathbb{P}(\mathrm{X}, \mathrm{Z}) \cup|\mathrm{Y}| \mathrm{K}_{1}$. Using Lemma 1 , we see that $\eta(G)=n-2$.

Theorem 8 Let $\mathrm{G} \in \mathcal{T}_{\mathrm{n}}$ with tripartition $(\mathrm{X}, \mathrm{Y}, \mathrm{Z})$ and $\mathrm{n} \geq 4$. Then $\mathrm{\eta}(\mathrm{G})=$ $\mathrm{n}-4$ if and only if $\mathrm{G}$ is a graph $\mathrm{H}$ possibly with some isolated vertices, where $\mathrm{H}$ is an expanded path of length 4 or the expanded graph $\mathbb{C}_{5}(\overline{\mathrm{e}})$.

Proof. Let $G \in \mathcal{T}_{n}$ with tripartition $(X, Y, Z)$ and $\eta(G)=n-4$. Let $A(G)$ be the adjacency matrix of $G$ defined by $(3)$. Then by $(8)$, we have $\operatorname{rank}(C)=1$, that is, $\operatorname{rank}(\mathrm{L})=1$. This means that there is only one independent row, say, $L_{y_{0}}$ in $L$, where $y_{0} \in Y$ is the vertex corresponding to $L_{y_{0}}$. Then for each $y \in Y$, either $N(y)=N\left(y_{0}\right)$ or $N(y)=\emptyset$. Let $Y_{1} \subseteq Y$ is the set of all vertices of $Y$ with non-zero degree. We have the following three cases.

Case 1. When $N_{Z}\left(Y_{1}\right)=\emptyset$. In this case, $N\left(Y_{1}\right) \subseteq X$. By condition $(1), N\left(Y_{1}\right) \neq$ $X$. We partition $X, Y$ and $Z$ into $Y_{1}, N\left(Y_{1}\right), Z$ and $X \backslash N\left(Y_{1}\right)$. Then $G$ can be drawn as an expanded path $\mathbb{P}\left(Y_{1}, N\left(Y_{1}\right), Z, X \backslash N\left(Y_{1}\right)\right)$ possibly with some isolated vertices in $Y \backslash Y_{1}$.

Case 2. When $N_{X}\left(Y_{1}\right)=\emptyset$. In this case, $N\left(Y_{1}\right) \subseteq Z$. By condition $(1), N\left(Y_{1}\right) \neq$ $Z$. We partition $X, Y$ and $Z$ into $Y_{1}, N\left(Y_{1}\right), X$ and $Z \backslash N\left(Y_{1}\right)$. Then $G$ can be drawn as an expanded path $\mathbb{P}\left(Y_{1}, N\left(Y_{1}\right), X, Z \backslash N\left(Y_{1}\right)\right)$ possibly with some isolated vertices in $Y \backslash Y_{1}$.

Case 3. When $N_{X}\left(Y_{1}\right) \neq \emptyset$ and $N_{Z}\left(Y_{1}\right) \neq \emptyset$. We can partition $X$ into $X_{1}$ and $X^{\prime}$, such that $X_{1}=N_{X}\left(Y_{1}\right)$ and $X^{\prime}=X \backslash X_{1}$. Similarly, we can partition $Z$ into $Z_{1}$ and $Z^{\prime}$, such that $Z_{1}=N_{Z}\left(Y_{1}\right)$ and $Z^{\prime}=Z \backslash Z_{1}$. Then, using condition (2), one can draw the graph $G$ as an expanded graph $\mathbb{C}_{5}(\bar{e})$ (shown in Figure 1). 
There are possibly some isolated vertices in $Y^{\prime}$, where $Y^{\prime}=Y \backslash Y_{1}$.

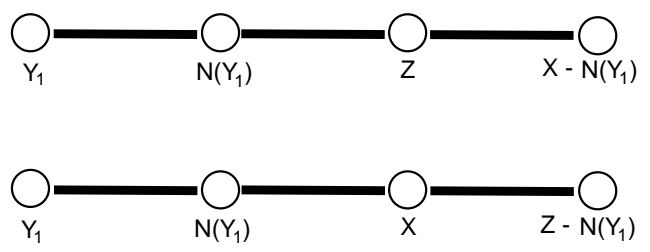

Figure 2: Two expanded paths $\mathbb{P}\left(\mathrm{Y}_{1}, \mathrm{~N}\left(\mathrm{Y}_{1}\right), \mathrm{Z}, \mathrm{X} \backslash \mathrm{N}\left(\mathrm{Y}_{1}\right)\right)$ and $\mathbb{P}\left(\mathrm{Y}_{1}, \mathrm{~N}\left(\mathrm{Y}_{1}\right), \mathrm{X}, \mathrm{Z} \backslash\right.$ $\left.\mathrm{N}\left(\mathrm{Y}_{1}\right)\right)$ of length 4

Conversely, let $\mathrm{G}$ be drawn as an expanded path of length 4 possibly with some isolated vertices. Then Lemma 1 yields that $\eta(G)=n-4$. Furthermore, if $\mathrm{G}$ can be drawn as $\mathbb{C}_{5}(\bar{e})$ with some isolated vertices, then using Lemma 5 , we get $\eta(G)=n-4$.

\section{Some graphs in $\mathcal{T}_{n}$ with nullity $n-6$}

In this section, we consider some graphs in $\mathcal{T}_{\mathfrak{n}}, \mathrm{n} \geq 6$, with nullity $n-6$. Let $\mathrm{G} \in \mathcal{T}_{\mathrm{n}}$ with tripartition $(X, Y, Z)$ and let $\mathrm{X}^{\prime}=\mathrm{X} \backslash \mathrm{N}_{\mathrm{X}}(\mathrm{Y})$. Note that $\mathrm{X}^{\prime} \neq \emptyset$ by (1). We assume that

$$
\mathrm{G}\left[\mathrm{N}_{\mathrm{X}}(\mathrm{Y}) \cup \mathrm{Y}\right]=\mathbb{P}\left(\mathrm{N}_{\mathrm{X}}(\mathrm{Y}), \mathrm{Y}\right)
$$

The following result gives a characterization of a graph $\mathrm{G}$ in $\mathcal{T}_{\mathrm{n}}, \mathrm{n} \geq 6$ satisfying (9) and $\eta(G)=n-6$.

Theorem 9 Let $\mathrm{G} \in \mathcal{T}_{\mathrm{n}}$ with tripartition $(\mathrm{X}, \mathrm{Y}, \mathrm{Z}), \mathrm{n} \geq 6$. Assume that $\mathrm{G}$ satisfies (9) and $\eta(\mathrm{G})=\mathrm{n}-6$. Then $\mathrm{G}$ has one of the following expanded decomposition.

(1) $\mathbb{C}_{5}(\bar{e}), \mathbb{P}_{2}$,

(2) $\mathbb{C}_{5}(\bar{e}), \mathbb{C}_{3}, \mathbb{P}_{2}$,

(3) $2 \mathbb{C}_{5}(\bar{e}), 2 \mathbb{P}_{2}$,

(4) $\mathbb{C}_{5}(\bar{e}), \mathbb{C}_{3}, 2 \mathbb{P}_{2}$ 
Proof. Let $G \in \mathcal{T}_{\mathfrak{n}}$ with tripartition $(X, Y, Z)$ satisfying $(9)$ and $\eta(G)=n-6$, $n \geq 6$. Let $X^{\prime}=X \backslash N_{X}(Y)$ and $Z^{\prime}=Z \backslash N_{Z}(Y)$. From (1), we see that $X^{\prime}$ and $Z^{\prime}$ are nonempty. Let $A(G)$ be the adjacency matrix of $G$ defined by (3). Since $\eta(G)=n-6$, using Corollary 4 , we have $\operatorname{rank}(\mathrm{L})=2$. This implies that $\mathrm{L}$ has two independent rows, say, $L_{y_{1}}$ and $L_{y_{2}}$, where $y_{1}, y_{2} \in Y$. Using (1) and (9), the columns of $L$ corresponding to the vertices of $X$ are constant. Then for each $y \in Y$, either $L_{y}=L_{y_{1}}$ or $L_{y}=L_{y_{2}}$. Thus we partition $Y$ into $Y_{1}$ and $\mathrm{Y}_{2}$, where

$$
\mathrm{Y}_{1}=\left\{\mathrm{y} \in \mathrm{Y} \mid \mathrm{L}_{\mathrm{y}}=\mathrm{L}_{\mathrm{y}_{1}}\right\}, \mathrm{Y}_{2}=\left\{\mathrm{y} \in \mathrm{Y} \mid \mathrm{L}_{\mathrm{y}}=\mathrm{L}_{\mathrm{y}_{2}}\right\}
$$

Note that $N_{Z}(y)=N_{Z}\left(Y_{1}\right)$ for each $y \in Y_{1}$, and $N_{Z}(y)=N_{Z}\left(Y_{2}\right)$ for each $y \in Y_{2}$. Since $\operatorname{rank}(L)=2$, either $N_{Z}\left(Y_{1}\right) \neq \emptyset$ or $N_{Z}\left(Y_{2}\right) \neq \emptyset$. Without loss of generality, assume that $N_{Z}\left(Y_{1}\right) \neq \emptyset$ and $N_{Z}\left(Y_{1}\right) \nsubseteq N_{Z}\left(Y_{2}\right)$. The following three cases are possible.

Case 1. When $N_{Z}\left(Y_{1}\right) \cap N_{Z}\left(Y_{2}\right)=\emptyset$.

If $N_{Z}\left(Y_{2}\right)=\emptyset$, then $N_{Z}(Y)=N_{Z}\left(Y_{1}\right)$. We draw the graph $G$ as an expanded graph on six expanded vertices $X_{1}=N_{X}(Y), X^{\prime}, Z_{1}=N_{Z}(Y), Z^{\prime}, Y_{1}$ and $Y_{2}$. Here $Y^{\prime}$ is possibly empty. The graph is shown in Figure 3 (i). In this case, we can decompose the graph $G$ into $\mathbb{C}_{5}(\bar{e})$ and $\mathbb{P}_{2}$.

If $N_{Z}\left(Y_{2}\right) \neq \emptyset$, we partition $N_{Z}(Y)$ into $Z_{1}=N_{Z}\left(Y_{1}\right)$ and $Z_{2}=N_{Z}\left(Y_{2}\right)$. We draw the graph $G$ as an expanded graph on seven expanded vertices $X_{1}=$ $N_{X}(Y), X^{\prime}, Z_{1}=N_{Z}\left(Y_{1}\right), Z_{2}=N_{Z}\left(Y_{2}\right), Z^{\prime}, Y_{1}$ and $Y_{2}$. The graph is shown in Figure 3 (ii). In this case, the graph can be decomposed into $\mathbb{C}_{5}(\bar{e}), \mathbb{C}_{3}$ and $\mathbb{P}_{2}$.

Case 2. When $N_{Z}\left(Y_{1}\right) \cap N_{Z}\left(Y_{2}\right) \neq \emptyset$ and $N_{Z}\left(Y_{2}\right) \nsubseteq N_{Z}\left(Y_{1}\right)$.

Let $Z_{1}=N_{Z}\left(Y_{1}\right) \cap N_{Z}\left(Y_{2}\right), Z_{2}=N_{Z}\left(Y_{1}\right) \backslash N_{Z}\left(Y_{2}\right)$ and $Z_{3}=N_{Z}\left(Y_{2}\right) \backslash N_{Z}\left(Y_{1}\right)$. Then $Z^{\prime}, Z_{1}, Z_{2}$ and $Z_{3}$ form a partition of $Z$. The graph can be drawn as an expanded graph on eight expanded vertices $X_{1}=N_{X}(Y), X^{\prime}, Z_{1}, Z_{2}, Z_{3}, Z^{\prime}$, $Y_{1}$ and $Y_{2}$. The graph is shown in Figure 4 (i). In this case, the graph $G$ can be decomposed into $2 \mathbb{C}_{5}(\bar{e})$ and $2 \mathbb{P}_{2}$.

Case 3. When $N_{Z}\left(Y_{1}\right) \cap N_{Z}\left(Y_{2}\right) \neq \emptyset$ and $N_{Z}\left(Y_{2}\right) \subseteq N_{Z}\left(Y_{1}\right)$.

We draw the graph $G$ as an expanded graph on seven expanded vertices $X_{1}=$ $N_{X}(Y), X^{\prime}, Z_{1}=N_{Z}\left(Y_{2}\right), Z_{2}=N_{Z}\left(Y_{1}\right) \backslash N_{Z}\left(Y_{2}\right), Z^{\prime}, Y_{1}$ and $Y_{2}$. The graph is shown in Figure 4 (ii). The graph $G$ can be decomposed into $\mathbb{C}_{5}(\bar{e}), \mathbb{C}_{3}$ and $2 \mathbb{P}_{2}$. 


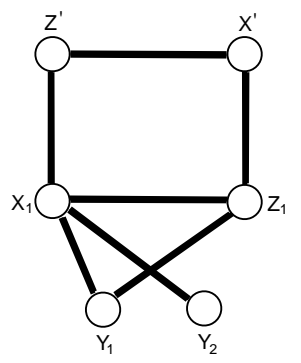

(i)

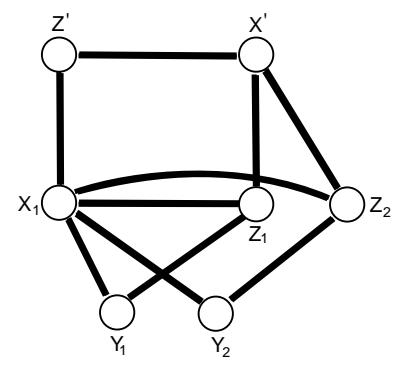

(ii)

Figure 3: Graphs drawn in Case 1 with $N\left(Y_{1}\right) \cap N\left(Y_{2}\right)=\emptyset$

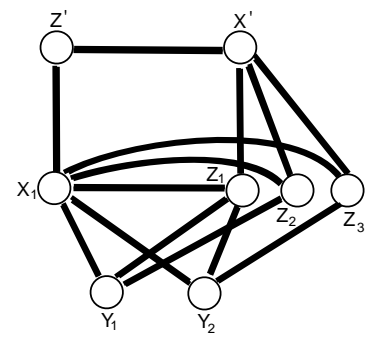

(i)

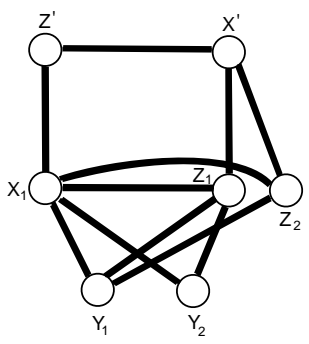

(ii)

Figure 4: Graphs drawn in Case 2 and Case 3 with $N\left(Y_{1}\right) \cap N\left(Y_{2}\right) \neq \emptyset$

\section{Conclusion}

We studied n-vertex tripartite graphs satisfying (1) and (2). We obtained the nullity set of this class of $n$-vertex tripartite graphs and characterized them with nullity $n-4$. It will be interesting to consider a more general class of n-vertex tripartite graphs and to characterize them with their nullity. In Theorem 9, we characterized those n-vertex tripartite graphs whose nullity is $\mathrm{n}-6$ and that satisfy (1), (2) and (9). We are not sure about the converse of Theorem 9 and it is left as an open problem. 


\section{References}

[1] B. Cheng, B. Liu, On the nullity of graphs, El. J. Lin. Algebra 16 (2007) 60-67. $\Rightarrow 99$

[2] L. Collatz, U. Sinogowitz, Spektren endlicher grafen, Abh. Math. Sere. Univ. Hamburg 21 (1957), 63-77. $\Rightarrow 97$

[3] Y.-Z. Fan, Yue Wang, Yi Wang, A note on the nullity of unicyclic signed graphs, Linear Algebra Appl. 438 (2013) 1193-1200. $\Rightarrow 97$

[4] Y.-Z. Fan, K.-S. Qian, On the nullity of bipartite graphs, Linear Algebra Appl. 430 (2009) 2943-2949. $\Rightarrow 97,99$

[5] S. Fiorini, I.Gutman, I. Sciriha, Trees with maximum nullity, Linear Algebra Appl. 397 (2005) 245-251. $\Rightarrow 97$

[6] S. Hu, T. Xuezhong, B. Liu, On the nullity of bicyclic graphs, Linear Algebra Appl. 429 (2008) 1387-1391. $\Rightarrow 97$

[7] W. Li, A. Chang, On the trees with maximum nullity, MATCH Commun. Math. Comput. Chem. 56, 3 (2006) 501-508. $\Rightarrow 97$

[8] W. Li, A. Chang, Describing the nonsingular unicyclic graph, J. Math. Study 4 (2007) 442-445. $\Rightarrow 97$

[9] J. Li, A. Chang, W.C. Shiu, On the nullity of bicyclic graphs, MATCH Commun. Math. Comput. Chem. 60, 1 (2008) 21-36. $\Rightarrow 97$

[10] S. Pirzada, An Introduction to Graph Theory, Universities Press, Orient BlackSwan, Hyderabad, India, 2005. $\Rightarrow 97$

[11] T. Xuezhong, B. Liu, On the nullity of unicyclic graphs, Linear Algebra Appl. 408 (2005), 212-220. $\Rightarrow 97$

Received: April 12, 2016 • Revised: May 30, 2016 